\title{
Conceptos básicos y métodos en geoarqueología: geomorfología, estratigrafía y sedimentología
}

\author{
Alfonso Benito-Calvo, Isidoro Campaña Lozano, \\ Theodoros Karampaglidis ${ }^{1}$
}

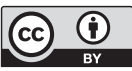

Recibido: 01-10-2014

Aceptado: 25-10-2014

\section{Resumen}

En este trabajo se sintetizan los conceptos y métodos básicos de tres de las ciencias más comunes en geoarqueología, como son la geomorfología, la estratigrafía y la sedimentología. A través de estas ciencias se trata de reconstruir los paisajes y los procesos de formación en torno a las ocupaciones humanas del registro arqueológico. Las distintas metodologías son de diversa naturaleza y escala, combinando técnicas cartográficas, principios geológicos, descripciones de campo y análisis de laboratorio para caracterizar los materiales y medios físicos asociados a las ocupaciones humanas.

Palabras clave: metodología básica; técnicas; geoarqueología.

Resum. Conceptes bàsics i mètodes en geoarqueologia: geomorfologia, estratigrafia i sedimentologia

En aquest treball se sintetitzen els conceptes i mètodes basics de tres de les ciències més comunes en geoarqueologia, com són la geomorfologia, l'estratigrafia i la sedimentologia. A través d'aquestes ciències es tracta de reconstruir els paisatges i els processos de formació al voltant de les ocupacions humanes del registre arqueològic. Les diferents metodologies són de diversa natura i escala, i combinen tècniques, principis geològics, descripcions de camp i anàlisis de laboratori per caracteritzar els materials i medis físics associats a les ocupacions humans.

Paraules clau: metodologia bàsica; tècniques; geoarqueologia.

Abstract. Basic concepts and methods in Geoarchaeology: Geomorphology, Stratigraphy and Sedimentology

In this paper, the basic concepts and methods of three of the most common sciences in Geoarchaeology (Geomorphology, Stratigraphy and Sedimentology) are summarized. Using these sciences, the landscape and the formation processes associated with the archaeological occupations are reconstructed. The different methodologies are diverse in nature and scale, combining

1. Centro Nacional de Investigación sobre la Evolución Humana. Paseo Sierra de Atapuerca, 3. 09002 Burgos, España. alfonso.benito@cenieh.es; isidoro.campanna@cenieh.es; theodoros.karampaglidis@cenieh.es 
mapping techniques, geological principles, field descriptions and laboratory analysis, in order to characterize the materials and physical environments related to archaeological sites.

Keywords: basic methodology; techniques; Geoarqueology.

Benito-Calvo, Alfonso; Campaña Lozano, Isidoro; Karampaglidis, Theodoros. «Conceptos básicos y métodos en Geoarqueología: Geomorfología, Estratigrafía y Sedimentología». Treballs d’Arqueologia, 2014, núm. 20, p. 41-54. DOI: 10.5565/rev/tda.45

\section{Introducción}

El desarrollo de los grupos de homínidos está estrechamente ligado al territorio o paisaje que habitan. Entre los numerosos componentes que definen el paisaje, los factores físicos del terreno constituyen el escenario en el cual interaccionan el resto de factores. El paisaje físico no es un medio invariable, sino que está caracterizado por constantes cambios a diversas escalas, que condicionan las características y los recursos de territorio, influyendo de manera notable en el desarrollo de las culturas y la formación y preservación de los registros arqueológicos. Las variaciones del territorio están determinadas a escalas generales por la tectónica de placas y la evolución geológica de las macroestructuras litosféricas (continentes, océanos, cordilleras, depresiones sedimentarias), y a escalas menores por la evolución de las meso y microestructuras del territorio donde se generan los yacimientos arqueológicos (costas, lagos, valles, llanuras, sierras, sistemas de cavidades, etc.). De este modo, la geoarqueología se ocupa del estudio de los componentes abióticos del paisaje, con objeto de reconstruir los paisajes físicos y los georrecursos utilizados por los grupos humanos, así como analizar los procesos de formación de los yacimientos y regis- tros arqueológicos. En este trabajo, se describirán brevemente los conceptos y las metodologías utilizados en tres de las ciencias más comunes en geoarqueología, como son la geomorfología, la estratigrafía y la sedimentología.

\section{Geomorfología}

El paisaje físico está articulado por un conjunto de morfologías, que, a grandes rasgos, pueden ser divididas en dos grandes grupos: formas erosivas y formas acumulativas. Las primeras son aquellas que se forman por la acción de los procesos erosivos y de alteración sobre el sustrato geológico, mientras que las segundas se generan por la acumulación de materiales, generalmente depósitos sedimentarios. Ambos tipos de morfologías se pueden estar formando en el presente, esto es, los procesos que las forman funcionan en la actualidad, o puede tratarse de morfologías heredadas que fueron generadas por procesos acaecidos en distintas etapas del pasado. El estudio combinado de la forma de dichas morfologías y su composición, textura y estructura (en el caso de las formas acumulativas), permiten interpretar su origen y los procesos que las formaron (fluviales, de gravedad, eólicos, kársticos, etc.). De este modo, si 
existe una secuencia de formas en el paisaje, pueden ser inferidos la sucesión de procesos y aproximar los ambientales en el pasado.

En función de estos conceptos, el análisis geomorfológico de un territorio aporta a la arqueopaleontología conocimientos fundamentales para una mejor interpretación de la génesis y el contexto temporal de las concentraciones arqueológicas y paleontológicas, a través de la compresión de las formas del paisaje donde se localizan y de los procesos que actúan en el presente y actuaron en el pasado. De igual modo, el relieve y los procesos geomorfológicos constituyen elementos clave para analizar la conservación y perduración de los yacimientos o concentraciones arqueopaleontológicas.

En el análisis geomorfológico se analizan las formas del paisaje (morfología, génesis y edad), y los procesos actuales, teniendo en cuenta las características del sustrato geológico y su estructura tectónica. Al tratarse de un estudio espacial, este análisis se sirve frecuentemente de cartografías y, a su vez, su producto final es expuesto comúnmente en modo cartográfico. Esta representación gráfica del paisaje es el mapa geomorfológico. La cartografía geomorfológica es un concepto relativamente reciente que surge a finales del siglo xIx y principios del siglo $\mathrm{xx}$ y tiene su mayor desarrollo a partir de la Segunda Guerra Mundial (ver Garzón, 1978; Peña, 1997a; MartínSerrano et al., 2004), en relación con grupos de trabajo europeos (Polonia, Francia, Holanda, Suiza, Checoslovaquia, Bélgica, Italia, Rusia, Alemania, Italia). Esta diversidad de escuelas y la variedad de enfoques aplicativos determinan que no exista una visión única de lo que debe entenderse por mapa geo- morfológico. Aunque han existido intentos de unificación en los sistemas cartográficos, las leyendas propuestas no han tenido una gran aceptación general, provocando la coexistencia de sistemas de representación internacionales, con las particulares de cada país y mezclas de ambos (Peña, 1997b). En cualquier caso, la cartografía geomorfológica debe proporcionar una información organizada y precisa de las formas del relieve y de los procesos geodinámicos (Martín-Serrano et al., 2004). En este sentido, los elementos a representar en un mapa geomorfológico son: información topográfica, hidrográfica, geológica y los datos morfológicos relativos a:

- Morfometría. Datos cuantitativos del relieve, como pendientes, orientaciones, alturas, etc.

- Morfografía. Descripción cualitativa respecto a la configuración geométrica de las formas.

- Morfogénesis. Referida a los procesos genéticos, sistemas morfogenéticos y agentes del modelado.

- Morfodinámica. Formas heredadas y funcionalidad de los procesos actuales.

- Morfocronología. Sucesión temporal de los grupos de formas y sedimentos correlativos; cronología relativa y absoluta.

La información topográfica y la hidrología principal son proporcionadas por los mapas topográficos de base, mientras que los datos geológicos (litología, estructura, formaciones superficiales o marco morfoestructural) pueden ser extraídos de mapas geológicos ya existentes. El mapa geomorfológico puede abarcar todos estos elementos morfológicos o centrarse en alguno de 
ellos, formando cartografías especiales, como cartografías morfoestructurales, morfométricas (mapas de pendientes, orientaciones, rugosidad, altitudes, etc.), o cartografías morfodinámicas (mapas de riesgos). Otras clasificaciones de los tipos de mapas geomorfológicos (Peña, 1997a), están en función de las características del relieve que abarcan (generales o parciales), del grado de aplicación del mapa (básicos o de investigación pura, aplicados y especiales), si tratan las formas individualmente o en conjunto (mapas de regiones geomorfológicas), dependiendo de su grado de elaboración, o en función de la escala (gran escala, escala media y pequeña escala, ver en Peña, 1997a).

El procedimiento para realizar el mapa constaría de una primera fase de búsqueda de los documentos básicos mencionados y de información bibliográfica, seguido de una fase posterior de realización del mapa (Martín-Serrano et al., 2004). En esta segunda fase se realiza el análisis de la información recopilada y la fotointerpretación de la zona, dando como resultado la elaboración de un mapa provisional que debe ser testado mediante el trabajo de campo. En el trabajo de campo se realiza el reconocimiento, descripción y representación de las morfologías, así como la descripción de las secuencias estratigráficas y litofacies de los depósitos y formaciones superficiales identificados, además de la toma de muestras si es necesario. La combinación sucesiva de los trabajos de fotointerpretación y reconocimiento de campo produce la elaboración definitiva de la cartografía geomorfológica. Tradicionalmente estos procesos se realizaban sobre formato papel, pero las facilidades que aporta actualmente la cartografía digital permi- ten su elaboración en formato digital, entrando en una tercera fase de elaboración consistente en la edición digital. En esta última fase se introducen los elementos espaciales y sus bases de datos asociadas en un Sistema de Información Geográfica (SIG) para su posterior representación, gestión y procesado.

La escala de los mapas geomorfológicos se debe elegir en función del propósito con el que se realiza el mapa, de la textura del relieve y de la información cartográfica preexistente de la región analizada. Una primera aproximación de la escala se puede realizar analizando la textura del relieve, referida a la densidad y el tamaño de las formas que presenta el terreno. Sin embargo, en la decisión final de la escala prevalecen los objetivos que persigue la cartografía. Dependiendo de las formas y procesos que se pretenden reflejar, será necesaria la elección de una escala adecuada para su representación, pudiéndose realizar a escalas pequeńas y medias $(<1: 100.000)$ la representación de regiones geomorfológicas o macroformas de gran extensión (pediplanos, relieves estructurales regionales, grandes edificios volcánicos, etc.), y a escalas grandes $(>1: 50.000)$, la representación de meso y microformas.

La realización de una cartografía geomorfológica y su grado de detalle dependen en gran medida de la información de soporte y análisis con la que se cuenta. Aunque existen diversas bases de datos espaciales que pueden aportar información de interés (Lozano y Sánchez, 1997), los documentos básicos de partida están constituidos por la topografía, las fotografías aéreas y el mapa geológico. El mapa topográfico proporciona la base planimetría y datos precisos y a escala sobre la orografía del área (curvas de nivel y cotas 

geomorfología, estratigrafía y sedimentología

singulares). Por otro lado, los mapas geológicos facilitan una primera inferencia de los procesos y formas del relieve a través del análisis de las unidades litológicas que componen el sustrato y de la estructura tectónica, pero, además, también pueden aportar una aproximación inicial a las formaciones cuaternarias, ya que suelen diferenciar los materiales holocenos y pleistocenos más representativos. Las fotografías aéreas representan uno de los documentos esenciales para la elaboración del mapa geomorfológico, puesto que proporcionan una imagen objetiva del territorio, básica para la fotointerpretación de las formas del relieve. Los vuelos fotogramétricos se organizan en pasadas paralelas que cubren el territorio y están formadas por fotogramas consecutivos y solapados, de tal modo que permiten la visión estereoscópica de la zona de solape. La interpretación de las formas del relieve a través de la visión estereoscópica, junto con el análisis de las texturas y tonalidades de las imágenes, permiten ir reconociendo los elementos geomorfológicos que componen el paisaje. En los últimos años, las bases de datos digitales han cobrado una importancia esencial en la elaboración de análisis geomorfológicos. Por ejemplo, imágenes de satélite, ortofotos o modelos digitales del terreno (MDT) proporcionan una representación espacial de distintas características del territorio que permiten tanto el análisis visual como el tratamiento numérico de los datos a través de distintos parámetros y algoritmos para la identificación de formas y procesos del paisaje (BenitoCalvo, 2013). Otros documentos que pueden aportar información de interés para la elaboración de la cartografía geomorfológica son los mapas edafológicos, de formaciones superficiales, de vegetación y usos del suelo, hidrogeológicos y/o tectónicos.

\section{Estratigrafía}

Estratigrafía es una ciencia geológica que trata de estudiar los diferentes estratos, sus interrelaciones espaciales-temporales y la interpretación genética de los materiales que componen dichos estratos. Su etimología viene del latín stratum y del griego grafia $(\gamma \rho \alpha \varphi i \alpha)$ cuyo significado literal es «la ciencia que trata de la descripción de las rocas estratificadas». Se basa en conocer en detalle las propiedades de las rocas, como su litología, su geometría y su disposición tridimensional, $y$, a partir de ello, comprender su génesis para fines científicos o aplicados (Vera, 1994).

La estratigrafía se basa en seis conceptos fundamentales:

1. Principio de la horizontalidad y continuidad lateral. Este principio establece que a priori un estrato tiene la misma edad a lo largo de toda su extensión horizontal y que considera como isócronas a las superficies de estratificación.

2. Principio de la horizontalidad original. La geometría de los estratos se dispone paralela a las superficies de deposición de forma horizontal o subhorizontal.

3. Principio de la superposición de estratos. Dada una sucesión de estratos, los estratos superiores serán más recientes que los inferiores. Existen casos donde no se cumple este criterio, por procesos postdeposicionales (erosión, deformación por disolución y colapsos) o por tectónica. 
4. Principio del uniformismo o actualis$m o$. Este principio establece que, a lo largo de la historia de la Tierra, los procesos han sido uniformes (uniformismo) y parecidos a los actuales (actualismo), es decir, que las leyes que rigen los procesos geológicos han sido las mismas y producen los mismos efectos durante toda la historia de la Tierra.

5. Principio de la sucesión faunistica o de la correlación. Consiste en aceptar que cada intervalo cronológico registrado en la Tierra y representado por distintos estratos contiene diferentes fósiles de diferentes épocas geológicas. Esto permite establecer correlaciones entre sedimentos y rocas de distinta composición y de diferentes contextos geográficos.

6. Principio de la sucesión de eventos. Consiste en reconocer estratos que contienen evidencias de eventos y sucesos geológicos de gran magnitud y que delimitan grandes divisiones geológicas (cambios climáticos, cambios del nivel del mar, explosiones de volcanes, terremotos, cambios de campo magnético terrestre, meteoritos, desaparición de especies, etc.).

Son diversas y múltiples las aplicaciones de la estratigrafía a la geoarqueología, que se pueden resumir en delimitar y ordenar las sucesivas unidades litoestratigráficas, correlacionar dichas unidades, posicionarlas en marcos temporales, y conocer su génesis y comprender su evolución a lo largo del tiempo.

A continuación, se van a describir brevemente los principales conceptos estratigráficos, básicos para la descripción de las secuencias estratigráficas en campo.

\section{a) El estrato}

El estrato es un nivel de roca con características y propiedades homogéneas o graduales formada en una etapa temporal de deposición determinada. Los rasgos principales que delimiten la geometría y la forma de un estrato es el muro, que es la parte inferior de un depósito, el techo que es la parte superior de dicho nivel y su espesor. Según su geometría tridimensional, se podía catalogar en estratos tabulares, con superficies de estratificación planas y paralelas; en estratos irregulares, con un muro constituido por superficies anómalas y discontinuas y el techo plano; en estratos acanalados, cuya forma se parece mucho a un canal; en estratos de forma de cuña; en estratos de forma lenticular, que tienen el muro plano y el techo convexo; y finalmente en estratos ondulados, con muro plano y techo ondulado (Vera, 1994).

Cuando los estratos se analizan como un conjunto de sucesión de niveles, se reconocen varios tipos descriptivos y se les dan los siguientes nombres: uniforme, cuando los estratos que componen la secuencia estratigráfica que queremos analizar tienen un espesor análogo; aleatorio, con espesores muy variados; creciente, cuando los espesores de los estratos se van creciendo hacia el techo; estratodecreciente, cuando los espesores se van disminuyendo hacia el techo, y en haces, cuando los estratos se encuentran en un mismo grupo y se van repitiendo.

\section{b) Facies}

Dicho término corresponde a la agrupación de un conjunto de estratos en que se observan semejantes propiedades y 
características (litología, textura, geometría, color, estructuras, fósiles y edad). Las clasificaciones más usadas son las facies detríticas (turbidíticas o fluviales; Miall, 1978; Walker, 1978; Pickering et al., 1989) y las facies carbonatadas (Wilson, 1975; Flugel, 1982).

\section{c) Continuidad y discontinuidad}

Estos dos conceptos representan la relación genética entre diferentes cuerpos estratigráficos que componen la sección estratigráfica. El término de la continuidad se aplica cuando entre los dos materiales no hay signos de interrupción sedimentaria, mientras que la discontinuidad se utiliza cuando se registran episodios que confirman que hubo signos de interrupción y cambio. En función de la geometría de la superficie de separación entre los cuerpos litoestratigráficos se pueden reconocer diversos tipos de discontinuidades. Se denomina paraconformidad cuando la geometría de la superficie del contacto es plana y paralela, mientras que se usa disconformidad cuando dicha superficie de separación es irregular y de carácter erosivo. Según la geometría de la superficie de disconformidad, se pueden reconocer diversas formas (discordancias angulares, discordancia angular erosiva, discordancia sintectónica e incorfomidad) (Vera, 1994).

Aplicando estos conceptos se realiza el levantamiento estratigráfico, el cual puede definirse como el registro y la documentación cronológica de los distintos sucesos sedimentarios en una secuencia. Puede tener carácter local, nivel regional (por ejemplo una cuenca) o nivel global. Cuando se trata de materiales superficiales, la toma de datos y el reconocimiento de las distintas unidades litoestratigráficas se hacen a partir de fotos aéreas, fotos de satélite, ortofotos, fotogrametría, escáner láser 3D, estación total, GPS decimétrico y, por supuesto, trabajo de campo. Pero cuando se trata de reconocer la litología del subsuelo, la toma de datos se puede hacer mediante sondeos (geológicos-arqueológicos), prospecciones geofísicas y diagrafías.

Con carácter local y aplicado a levantamientos arqueoestratigráficos, el avance tecnológico y el desarrollo de nuevas técnicas de documentación topográfica como la fotogrametría y el escáner láser 3D nos permiten capturar de manera discreta, pero a muy alta resolución, cualquier elemento en tres dimensiones (3D) y su textura fotográfica. De este modo, nuestro objetivo de estudio puede ser analizado digitalmente en entornos CAD, SIG o BD. El producto generado es una nube de puntos o una malla de triangulación de alta densidad en verdadera magnitud, a partir de la cual podremos realizar cálculos métricos, cartografiar unidades estratigráficas para realizar cortes o secciones, vectorizar todos los elementos reconocidos y combinarlos con datos extraídos de diferentes escalas (ortofotos, fotos de satélite para escalas pequeñas y estación total y GPS decimétrico para escalas grandes), de distintas campañas o de diverso origen (geológico, arqueológico o paleontológico).

\section{Sedimentología}

La sedimentología es la ciencia que estudia las rocas sedimentarias y los sedimentos, describiendo su composición, su textura y sus estructuras para entender los procesos de formación de las rocas sedimentarias. Las rocas sedimentarias se 
forman por el depósito de sedimentos generados a partir de un proceso de erosión de un área fuente, transporte por procesos geológicos y depósito en una cuenca sedimentaria.

La erosión implica la desaparición de la roca madre por procesos físicos, químicos y/o biológicos originando un residuo de partículas sólidas y/o productos químicos. Ejemplos de tipos de procesos erosivos importantes son cursos fluviales, procesos eólicos, ciclos de humedad-desecación, ciclos de frío-calor y disolución.

El residuo generado por la erosión puede ser transportado o no. Si no se produce transporte, se acumulan formando un suelo que puede quedar preservado en el registro geológico. La mayoría de los residuos son transportados hasta una zona de deposición. Hay varios procesos de transporte que dependen de las condiciones ambientales y geomorfológicas del lugar, aunque se pueden separar en dos grandes grupos: procesos de transporte en masa y procesos de transporte por fluidos. Los transportes en masa se producen por efecto de la gravedad, cuando la acumulación de sedimentos en una ladera supera un tamaño crítico, y deja de ser estable, colapsando y desplazándose hacia zonas más bajas. La presencia de agua puede ayudar al deslizamiento reduciendo la fricción en el sedimento, pero no es el responsable directo del transporte. Dentro de este grupo, dependiendo del tamaño de partícula y la cantidad de agua, se engloban desde caída de rocas, flujos de barros, hasta corrientes de turbidez. Los transportes por fluidos suponen el arrastre del material mediante flujos de agua o aire. Para que la partícula sedimentaria inicie el movimiento dentro del flujo es necesaria una energía inicial mayor que la necesaria para mantenerla en movimiento. El transporte por fluidos puede ser por tracción, saltación o suspensión. Por tracción la partícula se mueve por el fondo de la corriente, levantándose una distancia menor a su diámetro, deslizándose o rodando. Este tipo de transporte es el más común entre los tamańos de gravas y mayores. En el transporte por saltación la partícula se desplaza con contactos intermitentes con el suelo, moviéndose mediante una serie de saltos; comúnmente este es el transporte de la fracción arena del sedimento. Las partículas de menor tamaño se mueven por suspensión; en este caso, las partículas se mantienen en el flujo durante largas distancias, decantando solo cuando la energía del mismo disminuye.

El depósito del sedimento se produce cuando la energía del proceso sedimentario disminuye de tal manera que ya no es capaz de mantener en movimiento las partículas sedimentarias y estos caen al fondo. Los sedimentos se acumulan en zonas deprimidas llamadas cuencas sedimentarias. El estudio del relleno sedimentario de estas cuencas nos indica la evolución paleogeográfica de la región.

El estudio sedimentológico de una cuenca sedimentaria requiere un exhaustivo trabajo de campo y laboratorio donde se identifiquen las principales características de los depósitos sedimentarios y sus morfologías en la cuenca. Para ello es imprescindible hacer una buena descripción en campo de los depósitos sedimentarios identificados. En esta descripción deben quedar reflejadas las características más importantes de la roca sedimentaria como la litología, el color, la textura, la geometría o las estructuras sedimentarias. La textura 
sedimentaria de una roca sedimentaria se refiere a las características de tamaño, forma y orientación de las partículas sedimentarias que lo forman. Su estudio requiere descripciones de campo y medidas en laboratorio. Las estructuras sedimentarias son morfologías y formas distinguibles en las rocas sedimentarias, como por ejemplo ripples, laminación cruzada o laminación paralela. Tanto la textura como las estructuras sedimentarias están configuradas por los procesos de transporte y de depósito que haya tenido el sedimento. Una buena descripción nos permitirá hacer diferencias entre nuestros sedimentos y definir las facies sedimentarias de nuestra cuenca. El término facies es muy importante en sedimentología y se puede definir como un conjunto de rocas sedimentarias cuyas características texturales, estructurales, litológicas y geométricas nos permite diferenciarlas de otros conjuntos de rocas (Vera, 1994). Las facies sedimentarias se pueden relacionar con procesos sedimentarios.

Aunque las descripciones de campo son básicas para interpretar los sedimentos, no son suficientes para describir con precisión la textura, forma y composición de los sedimentos, especialmente en las partículas finas. Por ello, también se aplican distintas técnicas de laboratorio:

\section{Granulometría}

La granulometría es el estudio de la distribución de tamaño de partículas en un depósito sedimentario (Farrand, 2001). Las condiciones de transporte y depósito del sedimento es comúnmente inferido a partir de su distribución de tamaños de partículas (Clarke et al., 2014; Vandenberghe, 2013; Sánchez et al.,
2008; Powell, 1998; Middleton, 1976; Visher, 1969; Bagnold y Barndorff-Nielsen, 1980).

No existe una sola forma de medir el tamaño de una partícula. Las técnicas granulométricas usan valores diferentes para representar el tamaño de una partícula y todas están sesgadas por la forma de las partículas; por lo tanto hay que tener cuidado cuando se comparan datos obtenidos por técnicas diferentes, o al comparar muestras con formas de partículas muy diferentes.

El tamaño de partícula de un sedimento puede variar entre unas pocas micras hasta bloques de varios metros. Debido a esta variedad de tamaños, es más útil el uso de escalas logarítmicas o geométricas que lineales. La escala de tamaños usada en sedimentología es la Udden-Wentworth, propuesta en 1898 por Udden y modificada por Wentworth en 1922. En esta escala, cada valor es el doble que el valor inferior y la mitad que el valor superior. Para facilitar su uso, Krumbein propuso en 1934 la escala logarítmica phi, expresada por la siguiente fórmula:

$$
\Phi=-\log _{2} d
$$

Donde $\Phi$ es el valor de tamaño y $d$ el diámetro de la partícula en milímetros.

La granulometría de los tamaños gruesos se puede realizar mediante un contaje manual, mientras que, para las partículas finas, existen varias técnicas de laboratorio, entre las cuales destacan:

\section{Tamizado}

En la técnica del tamizado se hace pasar la muestra por unos tamices con una determinada abertura de malla, quedándose retenidas aquellas partículas mayo- 

geomorfología, estratigrafía y sedimentología

res a la luz del tamiz. De esta manera se consigue una división por tamańo de partícula en tantas clases texturales como tamices utilizados. El resultado se expresa en porcentaje en peso.

Los valores usados para los tamices dependen de la muestra y la cantidad de detalle que se busque. Es común usar tamices a intervalos de phi, $1 / 2$ phi o $1 / 4$ phi. Es importante usar el mismo intervalo de tamaño entre todos los tamices para obtener una distribución de tamaños correcta.

Los tamices consisten en una malla metálica formando aberturas cuadradas que discriminan según la sección menor de las partículas, es decir, la clasificación se hace por la anchura de la partícula. Debido a que la mayoría de las partículas no son esféricas, la capacidad para pasar por el tamiz depende de la correcta orientación de la partícula con respecto la abertura del tamiz. Cuanto más tiempo se tamice más probable es que la partícula obtenga esa orientación. Por ello es necesario usar las mismas condiciones de tiempo para todas las muestras. Por otro lado, la apertura cuadrada de la malla hace que la distancia máxima real de esta sea la diagonal del cuadrado, permitiendo el paso de ciertas partículas, aquellas cuya forma sean muy planas, con una anchura superior a la luz de malla (Sahu, 1964).

\section{Difracción láser}

La medición de tamaño de partículas por difracción láser se basa en la teoría de difracción de Fraunhofer (De Boer et al., 1987). Se basa en el principio de que las partículas difractan la luz en un ángulo que depende de su tamańo. En esta técnica se mide la difracción de un láser al incidir en las partículas con una orienta- ción aleatoria. El método permite medir un gran número de partículas en muy poco tiempo en un rango entre $0,4 \mu \mathrm{m} y$ $3000 \mu \mathrm{m}$.

La ventaja de la difracción láser radica en la poca cantidad de muestra requerida y en su velocidad para medir las muestras, en unos minutos se obtiene los valores granulométricos. Sin embargo, varios autores han mostrado variaciones en los resultados obtenidos por difracción láser y otras técnicas (Konert y Vandenberghe, 1997; Blott y Pye, 2006), advirtiendo de la dificultad para comparar esta técnica con otras.

\section{Tubos de decantación}

Hay diferentes análisis de tubos de decantación, pero todos se basan en el principio de que la velocidad de decantación depende del tamaño de la partícula. Para calcular el tamaño se usa la Ley de Stokes que relaciona la velocidad de caída de una partícula en un fluido con el tamaño de esa partícula. La decantación de la partícula depende también de la forma y la densidad; por ello el resultado se expresa en psi $(\psi)$, unidad de tamaño hidráulico equivalente, en vez de en phi. Esta técnica es usada para tamaños de partículas iguales o inferiores a arenas.

\section{Análisis de imagen}

El análisis de imagen consiste en la medición directa de las dimensiones sobre las imágenes de las partículas. Es una medición en dos dimensiones, normalmente realizada mediante un software que automáticamente identifica el límite de las partículas. La medición depende de la orientación de la partícula, aunque se acepta que la mayor parte de las partículas se apoyan sobre sus dos ejes mayores. Para una correcta medi- 
ción de cada partícula es necesario que se encuentren bien separadas entre sí. Aunque la separación puede hacerse manualmente, cuando se miden muchas partículas o muy pequeñas es necesario usar un dispersor.

Las medidas pueden realizarse sobre tamaños gruesos o tamaños finos. En los tamaños finos, las imágenes son obtenidas con un microscopio óptico, lo que implica que tiene las mismas limitaciones que el tipo de microscopio que se use. Por ello, no es una técnica apropiada para muestras muy heterogéneas, ya que es necesario tomar las imágenes con diferente distancia focal para enfocar las partículas.

Esta técnica presenta los resultados por número de partículas y un parámetro de tamaño como el eje mayor, el eje intermedio o el área. Para compararlos con otros métodos se expresa a veces el área como el diámetro equivalente de una esfera, que es el diámetro que tendría una esfera con el área de la partícula. $\mathrm{Al}$ ser mediciones directas de cada partícula, se pueden hacer tantas clasificaciones texturales como sean necesarias.

\section{Morfoscopia}

La morfoscopia consiste en la medición y el análisis de la forma externa de una partícula sedimentaria. La forma de las partículas se ha asociado con procesos de erosión y transporte sedimentario e influye en los análisis granulométricos.

Esta técnica usa imágenes de las partículas que luego son procesadas por software, separando el límite de la partícula del fondo. Una vez distinguida cada partícula, se miden índices morfológicos. Los índices medidos dependen del programa usado, pero en general relacionan el perímetro y el área de la partícula como aproximación a la forma de un círculo. Ejemplos de medidas tomadas en morfoscopia son la elongación, circularidad y convexidad.

\section{Análisis químicos}

Los análisis químicos nos permiten conocer las propiedades químicas de nuestro sedimento. Existen varios análisis químicos que se pueden hacer al sedimento, pero los más comunes aplicados a la geoarqueología son análisis de $\mathrm{pH}$, materia orgánica y carbonatos, y análisis mineralógicos y químicos.

\section{$p H$}

$\mathrm{El} \mathrm{pH}$ es un parámetro cuya interpretación no es directa, depende de varios factores como la humedad, la actividad orgánica y el sedimento. Los $\mathrm{pH}$ bajos pueden desencadenar una serie de reacciones químicas que alteren la composición inicial del sedimento y comprometa la conservación del registro fósil.

\section{Materia orgánica}

La cantidad de materia orgánica del suelo depende de la actividad biológica. La materia orgánica se puede medir mediante varios métodos, y el más usado es el método de ignición donde la muestra es calcinada a $550^{\circ} \mathrm{C}$. Valores altos de materia orgánica disminuyen el $\mathrm{pH}$ del sedimento.

\section{Carbonatos}

Debido a la sensibilidad de los carbonatos a medios ácidos, su porcentaje es un indicador de la intensidad de los procesos de alteración, o de la acción de procesos edáficos relacionados con carbonataciones. Un porcentaje alto de carbonatos 
sube el $\mathrm{pH}$ y previene que el valor disminuya por debajo de 7. Los carbonatos pueden ser calcita o dolomita, y se pueden formar en el sedimento o ser heredados. La dolomita tiene más resistencia al medio ácido.

\section{Análisis mineralógicos y composicionales}

La identificación de los minerales y elementos químicos que componen el sedimento es una herramienta muy útil para inferir los procesos de formación de los depósitos (sedimentarios y postdeposicionales) y su procedencia. Existen diversas técnicas y espectrometrías para conocer la composición de los sedimentos, entre las que destacan la difracción de rayos $\mathrm{X}$, la fluorescencia de rayos $\mathrm{X}$, la espectrometría Raman o la espectrometría de masas.

La difracción de rayos $\mathrm{X}$ es la técnica más usada para la identificación mineral en sedimentología. Permite el análisis cualitativo y cuantitativo de unos pocos gramos de muestra. Todos los minerales cristalinos son identificados con esta técnica, siempre que se encuentren suficientemente representados. Este método se basa en la difracción de los rayos X al incidir en un determinado ángulo sobre una estructura cristalina.
Por otro lado, la fluorescencia de rayos $\mathrm{X}$ determina la composición química de rocas y minerales. Es una técnica cuantitativa que permite el análisis de los elementos comprendidos entre el $\mathrm{F}$ y el $\mathrm{U}$, sobre sustancias sólidas. Se basa en la emisión secundaria de rayos $\mathrm{X}$ que se producen al excitar una muestra con una emisión primaria de rayos X. Esta emisión secundaria es característica para cada elemento químico.

La espectrometría Raman, tanto para las sustancias inorgánicas (minerales) como orgánicas del sedimento, es útil para aquellos compuestos cuya identificación no sea posible mediante la difracción de rayos $\mathrm{X}$ o cuando se quieren determinar sustancias orgánicas. El Raman hace incidir un haz de luz monocromático, como un láser, sobre un punto de la muestra. Este haz de luz es dispersado con ligeros cambios de frecuencia que son característicos de la estructura química del material analizado. Por otro lado, por medio de un espectrómetro de masas (ICP-MS), se consigue la detección de elementos muy precisos (ppb o parte por billón para la mayoría de los elementos), siendo muy adecuado para analizar los elementos traza de los sedimentos o partículas sedimentarias.

\section{Referencias bibliográficas}

Bagnold, R.A.; Barndorff-Nielsen, O. 1980. «The Pattern of Natural Size Distributions». Sedimentology 27: 199-207.

<http://dx.doi.org/10.1111/j.1365-3091.1980.tb01170.x>

Benito-Calvo, A. 2013. «La reconstrucción de los medios físicos y el análisis de paleopaisajes». En: García Díez, M.; Zapata, L. (eds.). Métodos y técnicas de análisis y estudio en arqueolo- 
gía prehistórica. De lo técnico a la reconstrucción de los grupos humanos: 119-140. Universidad del País Vasco, UPV/EHU.

Blott, S.J.; Pye, K. 2006. "Particle Size Distribution Analysis of Sand-Sized Particles by Laser Diffraction: An Experimental Investigation of Instrument Sensitivity and the Effects of Particle Shape». Sedimentology 53 (3): 671-85. <http://dx.doi.org/10.1111/j.1365-3091.2006.00786.x>

Clarke, D.W.; Boyle, J.F.; Chiverrell, R.C.; Lario, J.; Plater, A.J. 2014. "A Sediment Record of Barrier Estuary Behaviour at the Mesoscale: Interpreting High-Resolution Particle Size Analysis». Geomorphology 221 (0): 51-68. $<$ http://dx.doi.org/10.1016/j.geomorph.2014.05.029>

De Boer, G.B.; Cornelis de Weerd, J.; Thoenes, D.; Goossens, H.W.J. 1987. «Laser Diffraction Spectrometry: Fraunhofer Diffraction versus Mie Scattering». Particle Characterization 4 (1): 14-19. <http://dx.doi.org/10.1002/ppsc.19870040104>

FARRAND, W.R. 2001. "Archaeological Sediments in Rockshelters and Caves», in STEIN J.K.; Farrand, W. R. (eds). Sediments in Archaeological Context. Salt Lake City: The University of Utah Press.

Flugel, E. 1982. Microfacies analysis of limestones. Berlín: Springer-Verlag. <http://dx.doi.org/10.1007/978-3-642-68423-4>

Garzón, G. 1978. Metodología de la cartografía geomorfológica. Madrid: Fundación Juan March.

Konert, M.; Vandenberghe, J. 1997. «Comparison of Laser Grain Size Analysis with Pipette and Sieve Analysis: A Solution for the Underestimation of the Clay Fraction». Sedimentology 44 (3): 523-35. <http://dx.doi.org/10.1046/j.1365-3091.1997.d01-38.x>

Krumbein, W.C. 1938. «Size Frequency Distributions and the Normal Phi Curve». Journal of Sedimentary Petrology 8: 84-90. <http://dx.doi.org/10.1306/D4269008-2B26-11D7-8648000102C1865D>

Lozano, M. V.; SÁnChez, M. 1997. «Documentos básicos para la realización de mapas geomorfológicos». En: Peña, J.L. (ed.). Cartografía geomorfológica básica y aplicada: 25-48. Logroño: Geoforma.

Martín Serrano, A.; Salazar, A.; Nozal, F.; Suárez, A. 2004. Mapa geomorfológico de España a escala 1:50.000, guía para su elaboración. Madrid: IGME.

Mial, A.D. 1978. «Litofacies types and vertical profile models in braided river deposits: a summary». En: Mial, A.D. (ed.). Fluvial sedimentology, Can. Soc. Petrol. Geol. Mem., 5: $597-$ 604.

Middleton, G.V. 1976. «Hydraulic Interpretation of Sand Size Distributions». Journal of Geology 84: 405-26. <http://dx.doi.org/10.1086/628208>

PeñA, J.L. 1997a. «Los mapas geomorfológicos: características y tipos». En: PeÑA, J.L. (ed.). Cartografía geomorfológica básica y aplicada: 13-24. Logrońo: Geoforma.

- 1997b. «Los sistemas de cartografía geomorfológica». En: PeñA, J.L. (ed.). Cartografía geomorfológica básica y aplicada: 85-102. Logroño: Geoforma.

Pickering, K.T.; Hiscott, R.N.; Hein, F.J. 1989. Deep marine environments, clastic sedimentation and tectonics. Unwin \& Human, Inc., Wichester.

Powell, D. M. 1998. "Patterns and Processes of Sediment Sorting in Gravel-Bed Rivers». Progress in Physical Geography 22 (1): 1-32. <http://dx.doi.org/10.1177/030913339802200101>

SAHU, B. K. 1964. «Theory of sieving». Journal of Sedimentary Reserch 35(3): 750-53. <http://dx.doi.org/10.1306/74D71343-2B21-11D7-8648000102C1865D> 
Sánchez, A.; Álvarez-Legorreta, T.; Sáenz-Morales, R.; Ortiz-Hernández, M.; LópezOrtiz, B.E.; Aguíñiga, S. 2008. "Distribution of Textural Parameters of Surficial Sediments in the Bay of Chetumal: Implications for the Inference of Transport». Revista Mexicana de Ciencias Geológicas 25 (3): 523-32.

Udden, J. A. 1898. Mechanical composition of wind deposits. Augustana Library Pub. 1.

Vandenberghe, J. 2013. «Grain Size of Fine-Grained Windblown Sediment: A Powerful Proxy for Process Identification». Earth-Science Reviews 121: 18-30. <http://dx.doi.org/10.1016/j.earscirev.2013.03.001>

Vera, J.A. 1994. Estratigrafía: Principios y métodos. Madrid: Editorial Rueda.

Visher, G.S. 1969. «Grain Size Distribution and Depositional Processes». Journal of Sedimentary Petrology 39: 1074-1106.

WALKER, R.G. 1978. «Deep water sandstones facies and ancient submarine fans: models for exploration and for stratigraphic traps». Bull. Americ. Assoc. Petrol. Geol. 62: 932-66.

Wentworth, C.K. 1922. "A scale of grade and class terms for clastic sediments». Journal of Geology 30: 377-92. <http://dx.doi.org/10.1086/622910>

Wilson, J.L. 1975. Carbonate facies in Geologic History. Berlín: Springer-Verlag. <http://dx.doi.org/10.1007/978-1-4612-6383-8> 